In vitro intestinal co-culture cell model to evaluate intestinal absorption of edelfosine lipid nanoparticles

Running title: Caco-2/raji edelfosine lipid nanoparticles transport 


\title{
In vitro intestinal co-culture cell model to evaluate intestinal absorption of edelfosine lipid nanoparticles
}

\author{
Beatriz Lasa-Saracíbar ${ }^{1}$, Melissa Guada ${ }^{1}$, Victor Sebastián $^{2}$, Maria J. Blanco-Prieto ${ }^{1 *}$ \\ ${ }^{1}$ Department of Pharmacy and Pharmaceutical Technology, School of Pharmacy, University of Navarra, \\ Pamplona, Spain \\ ${ }^{2}$ Chemical \& Environmental Engineering Department \& Nanoscience Institute of Aragon (INA) \\ University of Zaragoza, Zaragoza, Spain
}

*Corresponding author: Dr. María J. Blanco-Prieto, Department of Pharmaceutics and Pharmaceutical Technology, School of Pharmacy, University of Navarra, C/Irunlarrea 1, E-31080 Pamplona, Spain, Office phone: + 34948425600 ext. 6519, Fax: + 34948425 649, e-mail: mjblanco@unav.es 


\begin{abstract}
Nanotechnology is providing a new therapeutic paradigm by enhancing drug efficacy and preventing side-effects. Edelfosine is a synthetic ether lipid analogue of platelet activating factor with high antitumor activity. The encapsulation of this potent antitumor drug in lipid nanoparticles increases its oral bioavailability; moreover, it prevents the hemolytic and gastrointestinal side-effects of the free drug. The literature points towards lymphatic absorption of lipid nanoparticles after oral administration, and previous in vitro and in vivo studies stress the protection against toxicity that these nanosystems provide. The present study is intended to assess the permeability of lipid nanoparticles across the intestinal barrier. Caco-2 monoculture and Caco-2/Raji co-culture were used as in vitro models of enterocytes and Microfold cells respectively. Results showed that free drug is internalized and possibly metabolized in enterocytes. These results do not correlate with those observed in vivo when edelfosine-lipid nanoparticles were administered orally in mice, which suggests that the microfold model is not a good model to study the absorption of edelfosine-lipid nanoparticles across the intestinal barrier in vitro.
\end{abstract}




\section{Keywords}

Caco-2, Edelfosine, Lipid nanoparticles, Permeabiltity, Raji, Transport 


\section{Introduction}

Edelfosine (ET) is a synthetic ether lipid analogue of platelet activating factor (PAF) with proved in vitro and in vivo antitumor activity [1]. However, it presents some drawbacks such as hemolysis after intravenous administration and gastrointestinal toxicity and poor bioavailability after oral administration [2]. These drawbacks can be overcome through nanotechnology [3]. Lipid nanoparticles (LN) prevent ET toxicity [4] and improve its oral bioavailability [2]. Moreover, they can be formulated by an organic solvent free method using biodegradable lipids [5]. Due to their physicochemical characteristics, LN containing ET (ET-LN) can be administered orally, intravenously and intraperitoneally [3]. Bearing in mind the importance of oncological patient well-being, the possibility of administering therapy by oral route is a challenge. Several in vivo studies report accumulation of the drug in the lymph nodes, endorsing lymphatic intestinal absorption after LN oral administration [3]. In order to obtain further insight into the evaluation of drug absorption in the gastrointestinal tract, several in vitro models have been developed since the 1980s [6]. Among all these models, Caco-2 monoculture is one of the most commonly used due to its ability to simulate the intestinal epithelium. The human epithelial colorectal adenocarcinoma cell line Caco-2 differentiates to enterocyte-like cells under specific culture conditions [6]. In addition, the hypothesis of lymphatic oral absorption of LN has promoted the development of intestinal in vitro models that simulate microfold cells (M cells) of Peyer's patches in the intestinal follicle-associated epithelium (FAE). $\mathrm{M}$ cells specialize in transporting soluble macromolecules, small particles and entire microorganisms from the intestinal lumen to the immune system. These cells have unique morphological features including the presence of a reduced glycocalyx, irregular brush border and reduced microvilli [7]. Previous authors have developed numerous strategies to obtain M-cell like in vitro models [8-12]. This work includes the development of an in vitro model of $\mathrm{M}$ cells based on the strategy of co-cultivating Caco-2 cells with Raji cells (B lymphocytes derived from Burkitt cell lymhoma) [9]. ET and ET-LN transport across mono and co-culture models are evaluated in the present study. 


\section{Material and methods}

\subsection{Materials}

ET was purchased from APOINTECH (Salamanca, Spain). Precirol® ATO 5 was a gift from Gattefossé (France). Tween ${ }^{\circledR} 80$ was purchased from Roig Pharma (Barcelona, Spain). Chloroform was obtained from Panreac (Madrid, Spain), formic acid 99\% for mass spectroscopy was obtained from Fluka (Barcelona, Spain), and methanol was purchased from Merck (Barcelona, Spain). All solvents employed for the chromatographic analysis were of analytical grade; all other chemicals were reagent grade and used without further purification. Amicon Ultra-15 10,000 MWCO centrifugal filter devices and Millicell Cell Culture Inserts were purchased from Millipore (Cork, Ireland). RPMI 1640 and MEM cell culture media, Heat-inactivated Fetal Bovine Serum (FBS), Glutamax, MEM Non-Essential Amino Acids, Penicillin/Streptomycin antibiotics, ZO-1 Monoclonal Antibody, Villin-1 Polyclonal Antibody, Alexa Fluor®594 Goat Anti-Rabbit IgG (H+L), Topro-3 Iodide and CellTrace CFSE Cell Proliferation Kit were purchased from Life Technologies, (Barcelona, Spain). DAPI was obtained from Invitrogen (Madrid, Spain). Fluorescence mounting medium was obtained from Dako (Barcelona, Spain). Rhodamine 123 and fluorescein sodium were obtained by Sigma Aldrich Química S.A. (Madrid, Spain). M-PER Mammalian Protein Extraction Reagent and Silicone tubing were purchased from Thermo Fisher Scientific (Madrid, Spain).

\subsection{Preparation and characterization of lipid nanopartciles}

LN were prepared by the hot homogenization method consisting of high shear homogenization and ultrasonication [13]. ET (30 mg) and Precirol® $(300 \mathrm{mg})$ were melted at approximately $5^{\circ} \mathrm{C}$ above the melting point of the lipid $\left(60^{\circ} \mathrm{C}\right)$. A $2 \%$ Tween ${ }^{\circledR} 80$ aqueous solution $(10 \mathrm{~mL})$ previously heated at the same temperature was added and dispersed in the molten lipid with the help of a Microson ${ }^{\mathrm{TM}}$ ultrasonic cell disruptor (NY, USA) for $1 \mathrm{~min}$ at an effective power of $10 \mathrm{~W}$. The preformed emulsion was then homogenized with an Ultraturrax ${ }^{\circledR}$ (IKA-Werke, Germany) for $1 \mathrm{~min}$ at 24,000 rpm and sonicated again with a Microson $^{\mathrm{TM}}$ ultrasonic cell disruptor (NY, USA) for $1 \mathrm{~min}$ at $10 \mathrm{~W}$. The emulsion was removed from heat and placed in an ice bath to obtain LN by lipid solidification. Then, the LN suspension was centrifuged using Amicon ${ }^{\circledR}$ Ultra-15 10,000 MWCO filters at $4500 \times \mathrm{g}$ for $30 \mathrm{~min}$ and washed twice with distilled water. Afterwards, $150 \%$ (w/w of lipid weight) trehalose was added as cryoprotectant agent to the LN suspension, which was then kept at $-80^{\circ} \mathrm{C}$ and freeze-dried to obtain a nanoparticulate powder. 
Particle size and polydispersity index (PDI) were evaluated by photon correlation spectroscopy (PCS) using a Zetasizer Nano (Malvern Instruments, UK). The measurements were carried out three times. Surface charge was measured using the same Zetasizer Nano equipment combined with laser Doppler velocimetry. For the ET loading determination, $5 \mathrm{mg}$ of nanoparticles were dissolved in $1 \mathrm{ml}$ of chloroform and mixed with $4 \mathrm{ml}$ of methanol. The mixture was vortexed for $1 \mathrm{~min}$ and then centrifuged at $20,000 \times g$ for $10 \mathrm{~min}$. The supernatant was analyzed by a previously validated ultra-high-performance liquid chromatography tandem mass spectrometry (UHPLC-MS/MS) method [14].

ET-LN morphology was evaluated by transmission electron microscopy (TEM). Images were taken on a FEI Tecnai T20 microscope at INA-LMA(Zaragoza). To prepare ET-LN samples for TEM observation, lyophilized NPs were dispersed in milli-Q water. After $30 \mathrm{~s}$ in an ultrasonic bath, a drop of this suspension was applied to a copper grid (200 mesh) coated with carbon film, and allowed to dry in air. The microscope was operated at $80 \mathrm{kV}$ to preserve the ET-LN morphology and diminish radiation damage.

\subsection{Cell culture}

Caco-2 cells were culture in MEM supplemented with $20 \%(\mathrm{v} / \mathrm{v})$ fetal bovine serum, $1 \% \mathrm{v} / \mathrm{v}$ nonessential aminoacids and $1 \%(\mathrm{v} / \mathrm{v})$ Glutamax at $37^{\circ} \mathrm{C}$ under a $5 \% \mathrm{CO}_{2}$ water saturated atmosphere. Cells were harvested with trypsin/EDTA every 3-4 days and subcultured at $12 \times 10^{3}$ cells $/ \mathrm{cm}^{2}$. Raji cells were cultured at $0.5 \times 10^{6}$ cells $/ \mathrm{ml}$ in RPMI supplemented with $20 \%(\mathrm{v} / \mathrm{v})$ fetal bovine serum, and $1 \%(\mathrm{v} / \mathrm{v})$ penicillin and streptomycin, at $37^{\circ} \mathrm{C}$ in a humid atmosphere with $5 \% \mathrm{CO}_{2}$. Cells were split 1:3-1:5 every 3-4 days.

\subsection{Enterocyte and M-cell like: in vitro models of gastrointestinal barrier}

\subsubsection{Development}

In vitro models were developed following a previous experiment by des Rieux et al. [9]. The human colon adenocarcinoma cell line Caco- 2 cells were seeded at a density of $9 \times 10^{4}$ cells on the apical chamber of Transwell inserts (3-mm pore PET Transwell filters; 12-well) in MEM supplemented with $20 \%(\mathrm{v} / \mathrm{v})$ fetal bovine serum, $1 \% \mathrm{v} / \mathrm{v}$ non-essential aminoacids, $1 \%(\mathrm{v} / \mathrm{v})$ Glutamax and 1\% penicillin and streptomycin. Cultures were maintained at $37^{\circ} \mathrm{C}, 5 \% \mathrm{CO}_{2}$ for up to 21 days. The medium was replaced every 2-3 days ( $0.5 \mathrm{ml}$ in the apical compartment and $1.5 \mathrm{ml}$ in the basolateral compartment). In 
case of the M-cell in vitro model, inserts were inverted after 14 days of culture and a piece of silicon (12.8 mm diameter) was placed on the basolateral side of each insert. The silicon piece was filled with a suspension of $5 \times 10^{5}$ Raji cells in $1 \mathrm{ml}$ of supplemented MEM. Co-culture was maintained for 4 days. Afterwards, Raji cells and silicon tubes were removed and inserts were placed in their original orientation. Transport experiments in both models were performed after 21 days of Caco- 2 seeding.

\subsubsection{Assessment of cell monolayer integrity}

Cell monolayer integrity was assessed in both models by measurement of trans-epithelial electrical resistance (TEER) [15]. Prior to measuring TEER, culture media was replaced by MEM without supplements. Inserts were placed at room temperature for 15 minutes and TEER was measured with a Millicell ERS-2 Voltohmmeter (Merck Millipore, Germany). The resistance is expressed as $\Omega / \mathrm{cm}^{2}$. Cell monolayer confluence was analyzed weekly. TEER measurement was also used to assess cell monolayer integrity during permeation experiments.

\subsubsection{Characterization of the in vitro intestinal models}

\subsubsection{Inmunofluorescence}

After 21 days of culture, cell monolayers were washed carefully with PBS, fixed with 4\% paraformaldehyde (w/v) and permeabilized with Triton X-100 $0.5 \%(\mathrm{v} / \mathrm{v})$. Monolayers were blocked with a 1\% BSA solution in TBS. Then cells were incubated with Anti-ZO-1 Alexa Fluor 488 (1:100) and Anti-villin (1:100) antibodies in TBS 1\%BSA o.n. Later, cells labelled with Villin antibody were incubated with Alexa Fluor ${ }^{\circledR} 594$ Goat Anti-Rabbit IgG (H+L) (1:500). Samples were stained with Topro3 Iodide or Dapi and visualized under fluorescence and confocal microscopy.

\subsubsection{Carboxyfluorescein succinimidyl ester (CFSE) labeling}

Raji cells were incubated with CellTrace CFSE for 15 minutes at $37{ }^{\circ} \mathrm{C}(10 \mu \mathrm{M}$ for flow cytometry and 25 $\mu \mathrm{M}$ for fluorescence microscopy). Afterwards, cells were incubated in culture media for 30 minutes and washed twice with PBS. Three days later, Caco-2 monolayers were co-cultured with the stained Raji cells as described above (section 2.4.1). After 21 days, cells monolayers were observed under fluorescence and confocal microscopy. Cells were fixed with 4\% paraformaldehyde and nuclei were stained with Topro-3 Iodide or Dapi. In addition, the presence of Raji cells in the culture was quantified by flow cytometry. 


\subsubsection{Transport studies}

TEER was measured before and after the experiments. Only cell monolayers with TEER values over 280 $\Omega / \mathrm{cm}^{2}$ were used. All transport studies were conducted under agitation at $37^{\circ} \mathrm{C}$ in transport medium (MEM without supplements). Total experiment volumes were $0.5 \mathrm{ml}$ in the apical compartment and 0.6 $\mathrm{ml}$ in the basolateral compartment. The different treatments were added to the donor compartments: Free ET (ET) and LN containing ET (ET-LN) at a final dose equivalent to $30 \mu \mathrm{g} / \mathrm{ml}$ of free drug. ET transport was studied from the apical compartment to the basolateral compartment $(A \rightarrow B)$ and vice versa $(B \rightarrow A)$. Fluorescein and rhodamine 123 were used as control substances in the transport experiments. Low permeable substance fluorescein free acid was added to the apical compartment at a final concentration of $100 \mu \mathrm{g} / \mathrm{ml}$. The p-glycoprotein substrate rhodamine 123 was added to the apical compartment $(\mathrm{A} \rightarrow \mathrm{B})$ and to the basolateral compartment $(B \rightarrow A)$ at a concentration of $5 \mu \mathrm{g} / \mathrm{ml}$. The experiment lasted 2 hours and donor samples were collected at the beginning and at the end of the experiment. Samples were analyzed using UHPLC-MS/MS [14] (ET and ET-LN) and fluorescence spectrometry (fluorescein and rhodamine). The permeability (apparent permeability coefficient; $\mathrm{P}_{\text {app }}$ ) was calculated according to the following equation [16]:

$\mathrm{P}_{\text {app }}=\mathrm{dQ} / \mathrm{dt} * 1 / \mathrm{A} * \mathrm{C}_{0}[\mathrm{~cm} / \mathrm{s}]$

' $\mathrm{dQ} / \mathrm{dt}$ ' is the rate of appearance of drugs on the acceptor compartment ( $\mu \mathrm{mol} / \mathrm{s}) ; \mathrm{C}_{0}$ is the initial drug concentration on the donor side $(\mathrm{mM})$; ' $\mathrm{A}$ ' is the surface area of the monolayer $\left(\mathrm{cm}^{2}\right)$.

\subsection{Statistical analysis}

Data analysis and graphic presentations were done using Prism version 5.00 software for Windows (GraphPad Software, San Diego, CA). Data are presented as a mean of three or more independent experiments, with error bars indicating the standard deviation. Data was analysed using a two-way ANOVA statistical test. The statistical significance level was defined as a $\mathrm{P}$ value of $<0.05$.

\section{Results and discussion}

\subsection{Lipid nanoparticle formulation}

The hot homogenization method consisting of high shear homogenization and ultrasonication provided LN with a size of $127.89 \pm 9.95 \mathrm{~nm}$ suitable for oral, intravenous and intraperitoneal administration [3] 
and low polidispersity index $(0.24 \pm 0.01)$. $\mathrm{LN}$ charge was negative and enough to maintain formulation stability $(-28.42 \pm 1.39 \mathrm{mV})$. This method of LN formulation has been previously developed and used by our research group $[13,17,18]$. The formulation used in this study was slightly modified in order to increase drug loading capacity. ET-LN loading was $22.677 \pm 2.262 \mu \mathrm{g}$ ET/mg formulation. In comparison to LN prepared in the above-mentioned previous studies, increasing the amount of drug that was initially added to the nanoparticles (from 15 to $30 \mathrm{mg}$ ) allowed us to obtain a higher loading capacity (from $13 \mu \mathrm{g}$ $\mathrm{ET} / \mathrm{mg}$ formulation to $22.677 \mu \mathrm{g} \mathrm{ET} / \mathrm{mg}$ of formulation). Despite the increase in drug loading capacity, ET-LN maintained their homogeneous morphology and round shape as is shown in TEM images (Fig. 1).
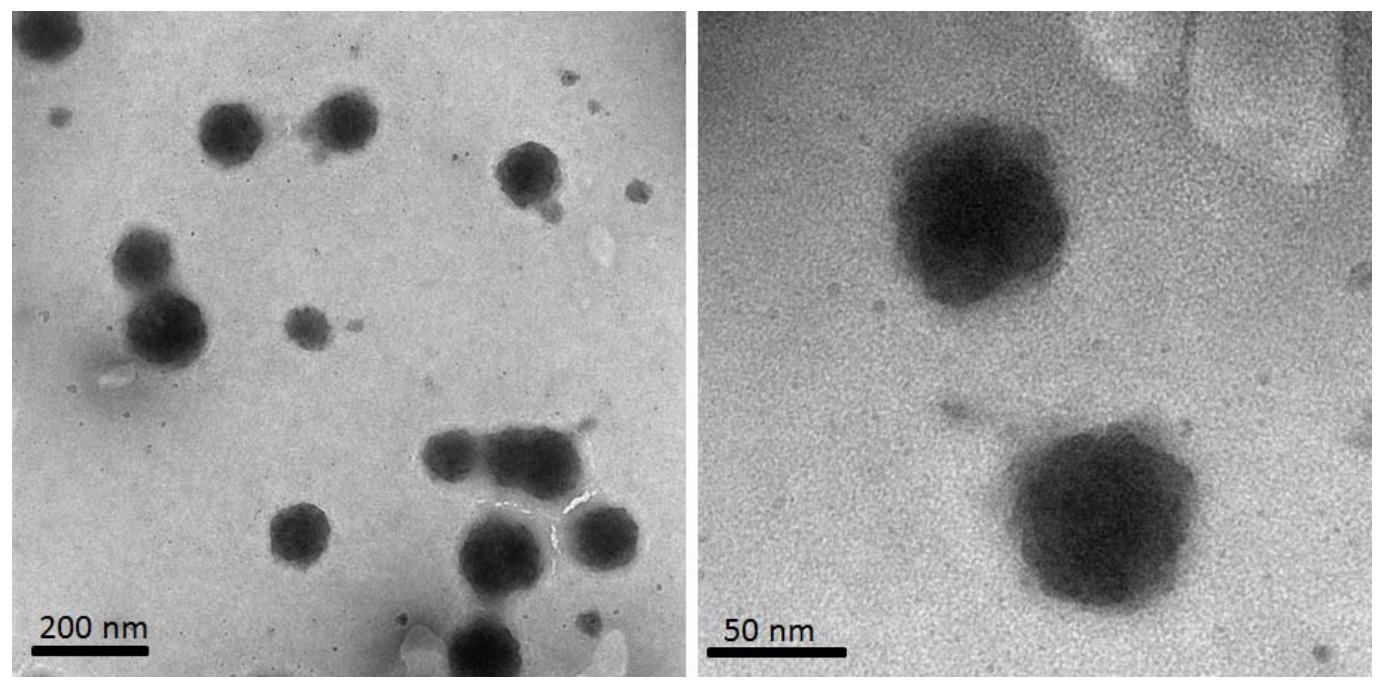

Figure 1. TEM images of LN containing edelfosine at different magnifications. ET-LN nanoparticles are not aggregated, which is in agreement with the polydispersity index obtained by PCS characterization.

\subsection{Transport experiments}

Several adapted in vitro models based on colorectal adenocarcinoma Caco-2 cells have been developed in the last decade to test drug permeability across the intestinal barrier [8, 9, 16, 19]. Among all these models, Caco-2 monoculture and co-culture of Caco-2 cells with Burkitt lymphoma B cells (Raji cells) are being widely used to simulate enterocytes and intestinal $\mathrm{M}$ cells respectively. As many researchers have reported significant accumulation of LN in the lymphatic system after oral administration [3], M-cell in vitro models have been developed in order to assess the absorption of these nano-systems by this route and compare it with the absorption by enterocytes (mono-culture of Caco- 2 cells). 
3.2.1. In vitro models of intestinal barrier: characterization

\subsubsection{Cell monolayer integrity}

Caco-2 monoculture and Caco-2/Raji co-culture presented similar TEER values after 21 days of culture (Fig. 2). As previously described, TEER values decreased after the co-culture with Raji cells for four days [9]. However, our results showed that, after removing Raji cells from the culture, TEER values recovered and achieved the monoculture values at day 21 (Fig. 2). Caco-2 monoculture and Caco-2/Raji co-culture showed TEER values of $480.2 \pm 11.56 \Omega / \mathrm{cm}^{2}$ and $502.8 \pm 23.50 \Omega / \mathrm{cm}^{2}$ respectively with no statistically significant differences between them. All monolayers reached TEER values above $200 \Omega / \mathrm{cm}^{2}$ at the time of the transport experiment, indicating the formation of a cell monolayer with maintenance of tight junction integrity [16].

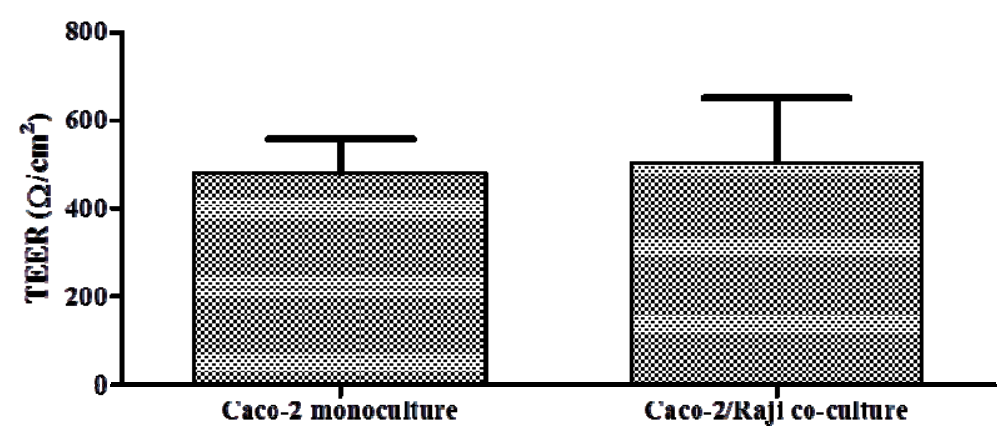

Figure 2. TEER values of Caco-2 monoculture and Caco-2/Raji co-culture after 21 days of incubation $\left(\Omega / \mathrm{cm}^{2}\right)$. Monoculture of Caco-2 and co-culture with Raji cells did not show differences in TEER values.

\subsubsection{Immunofluorescence characterization}

The formation of a monolayer containing cells phenotypically similar to enterocytes and $\mathrm{M}$ cells was monitored by immunofluorescence. Tight junctions were labeled with ZO-1 antibody [20]. ZO-1 is a protein that is present in the cytoplasmic membrane of cells with tight intercellular junctions. Both intestinal models showed the presence of this protein (Fig. 3). However, Caco-2 monoculture presented a more homogenous and structured monolayer architecture than Caco-2/Raji co-culture, indicating the formation of M-cells (Fig. 3). 

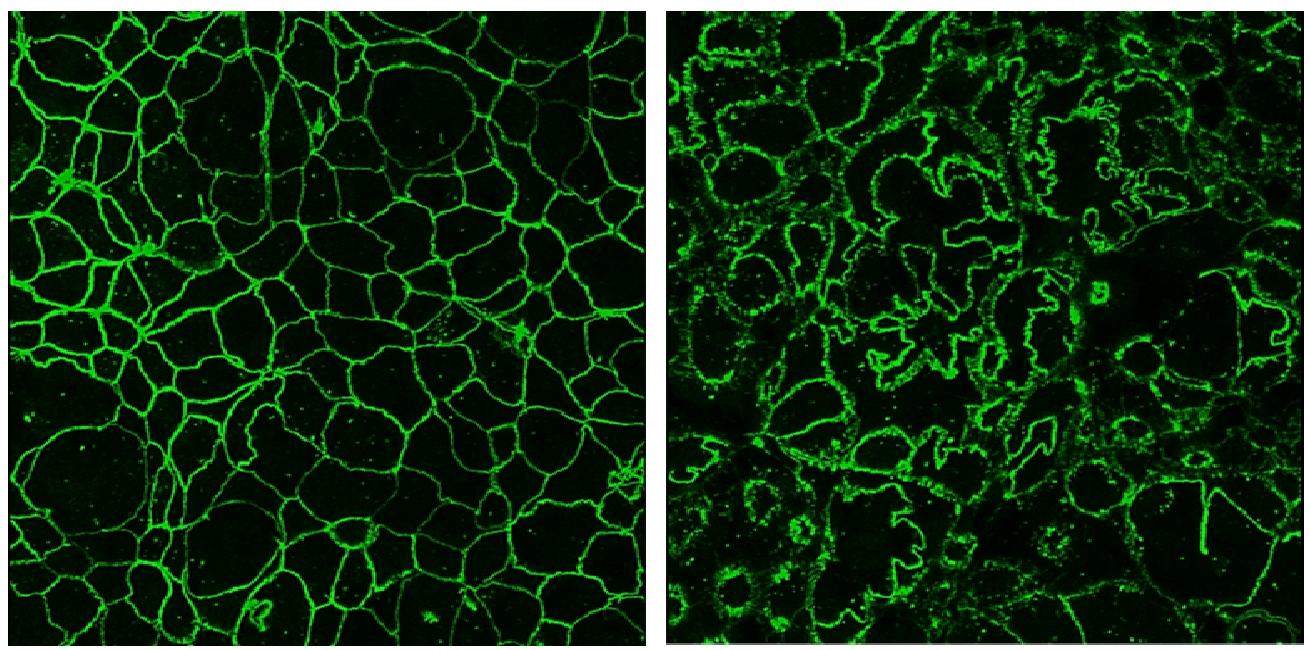

Figure 3. Confocal images of Caco-2 monoculture (left) and Caco-2/Raji co-culture (right) stained with ZO-1 antibody; $\mathbf{x 6 3 / 1 . 4 .}$

Presence of villin, a component of brush border assembly, was also assessed in both cultures. M cells are characterized by an irregular brush border and reduced microvilli to facilitate contact with particles and microorganisms [7].
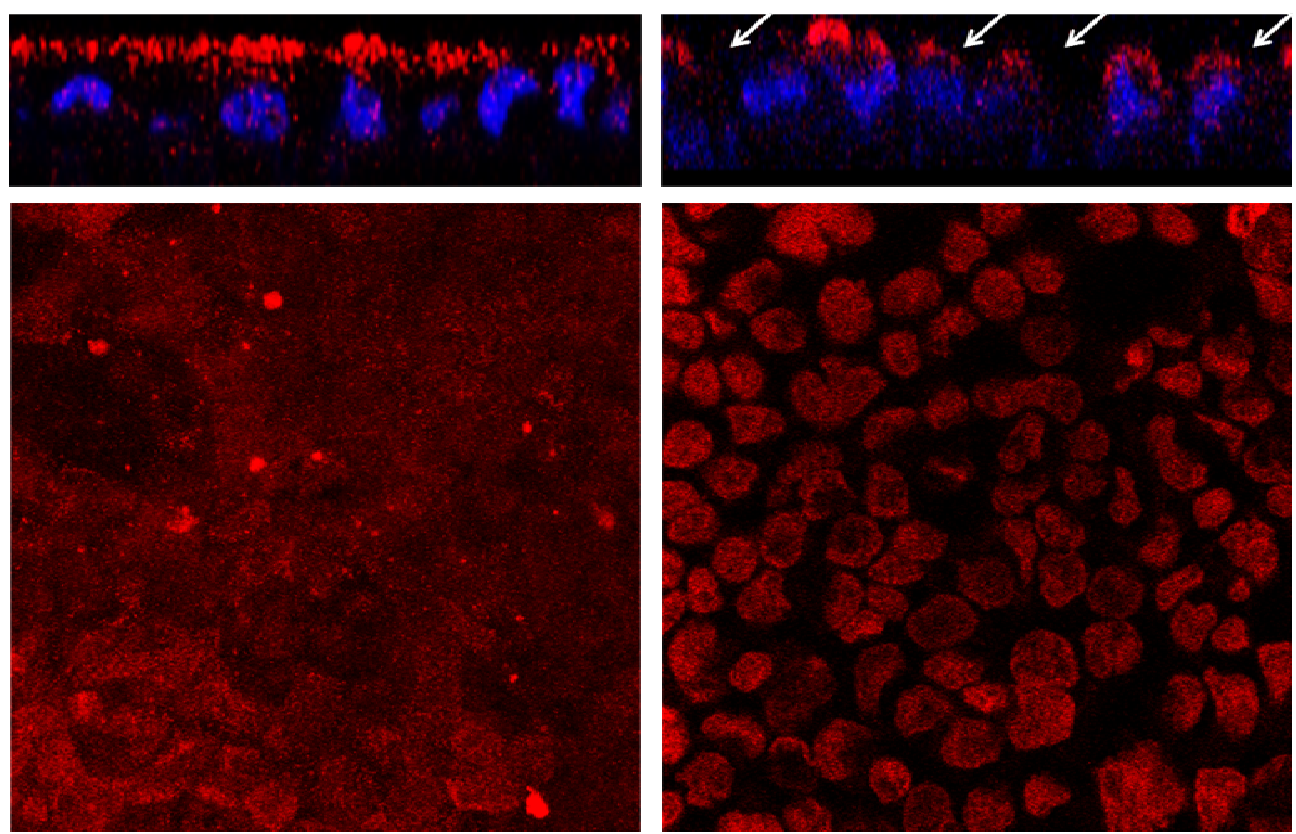

Figure 4. Lateral (upper figures) and frontal (lower figures) confocal images of Caco-2 monoculture (left) and Caco-2/Raji co-culture (right) stained with Villin antibody (red) and Topro-3 Iodide (blue); x63/1.4. Caco-2 monoculture showed a homogeneous layer of villin on the cell surface whereas it was partially distributed in the cell surface of the co-culture model (arrows indicate the lack of villin between cells).

Conversely, villin is present in $\mathrm{M}$ cells but its distribution is different, being located mainly in the cytoplasmic region [11, 21]. Such expression patterns were reproduced in our experiments (Fig. 4). Fig. 4 
shows that Caco-2 monocultures presented a homogenous layer of villin on the surface of the cells, indicating the development of a brush border typically present in enterocytes. Villin was also visible in Caco-2/Raji co-cultures but with a different distribution pattern; in this culture, some cells expressed villin in their surface but the protein did not cover the cell monolayer. The imperfect layer of villin indicates the differentiation of some Caco-2 cells into M-like cells with their typical reduced brush border.

\subsubsection{Localization and quantification of Raji cells in Caco-2/Raji co-culture (CFSE labeling)}

In order to localize Raji cells, we stained them with carboxyfluorescein diacetate succinimidyl ester (CFSE). This reagent passively diffuses into cells and turns into a fluorescent compound that is retained in the cell [22]. The developed model of co-culture is based on the direct contact of Raji cells with Caco-2 cells. At day 14, inserts were inverted and Raji cells were placed in the basolateral side of the membrane. Most authors support the need for direct contact between both cell lines in order to obtain a more reliable M-cell model $[9,11]$. In the inverted culture model, Raji cells are expected to migrate through the pores of the insert membrane and, therefore, to be incorporated into the Caco-2 cell monolayer. Presence of Raji cells within the monolayer was visualized by fluorescence microscopy and quantified by flow cytometry. The percentage of Raji cells in the culture assessed with flow cytometry was about $27 \%$ of all cells; these data were consistent with the results published by Rieux et al. [9], who obtained a percentage between 17 and $30 \%$ of total number of cells. Confocal images showed presence of CFSE labeled cells below the inserts (Fig. 5). Raji cells were not detected within the monolayer by fluorescence microscopy and the total proportion of fluorescent cells observable by fluorescence microscopy appeared to be lower than $30 \%$. This discrepancy might be explained due to the higher sensitivity of flow cytometry technique. Besides, Raji cells integrated into the cell monolayer might display lower fluorescence intensity, only detectable by flow cytometry. In conclusion, enterocyte and M-cell like in vitro culture models were successfully developed in our laboratory [9]. 

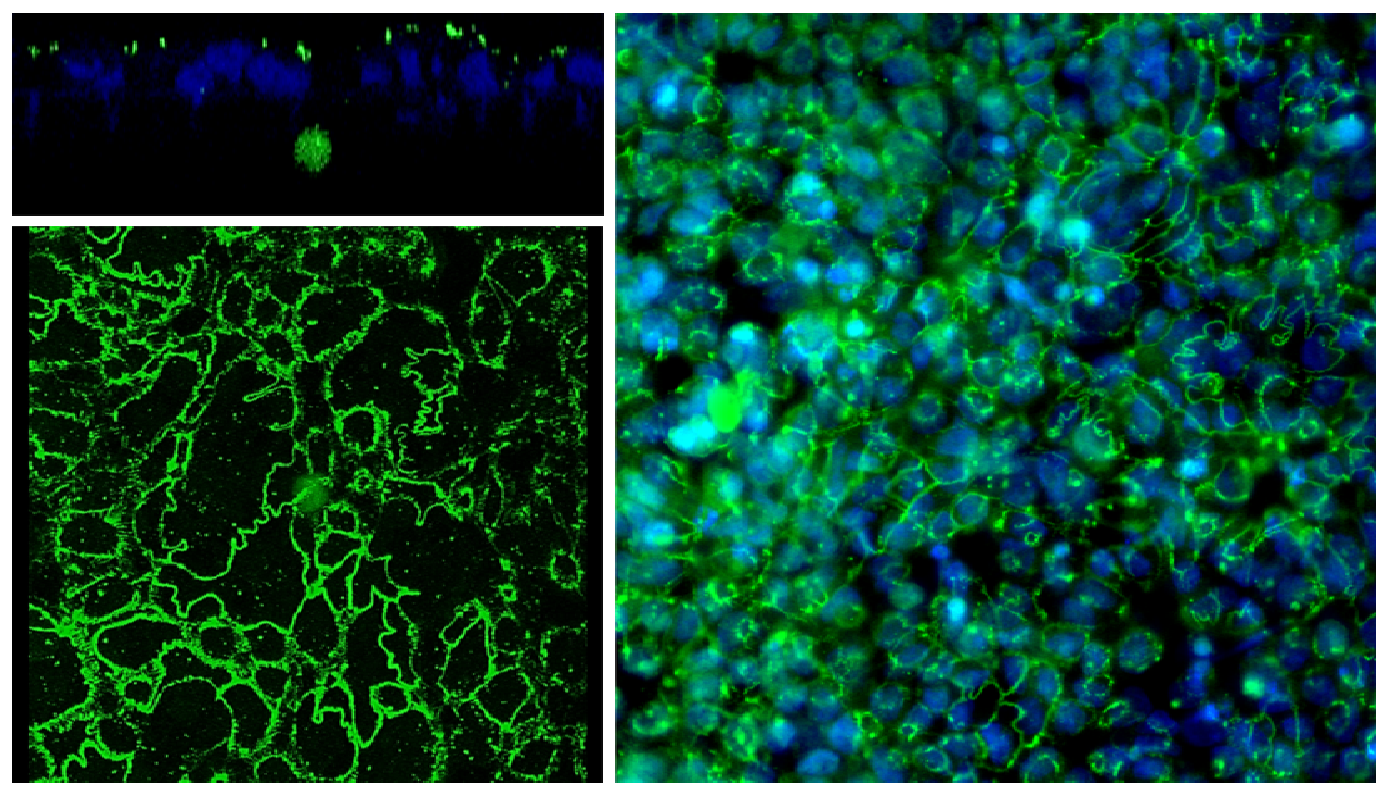

Figure 5. Lateral and frontal confocal images (upper and lower left) and fluorescence microscopy image (right) of Caco2/Raji co-culture. Tight junctions are stained with ZO-1 antibody (green) and nuclei are labelled with Topro-3 Iodide (blue); x63/1.4. Raji cells were stained with CFSE (green) prior to incubation with Caco-2 cells. Raji cells were detectable below the inserts.

\subsubsection{Transport studies}

Transport studies were performed in order to compare the transport of free drug and LN containing ET in the Caco-2 monoculture and Caco-2/Raji co-culture. Integrity of the cell monolayer was evaluated by measuring TEER values before and after transport experiments. TEER values showed that LN did not alter tight junctions; in contrast, ET produced a significant decrease of the TEER if it is incubated for four hours. Fig. 6 shows the changes in TEER values after two hours of incubation with the different treatments. As can be observed, free ET was the only treatment that produced a decrease in the transepithelial resistance. Our results led us to conclude that transport experiments could not last for more than two hours due to the high disruption of the cell culture, measurable by the TEER value, which was caused by the free drug. Although LN were not harmful for the insert cultures over longer times, transport experiments were performed up to 2 hours in order to compare ET and ET-LN transport in both in vitro models. 

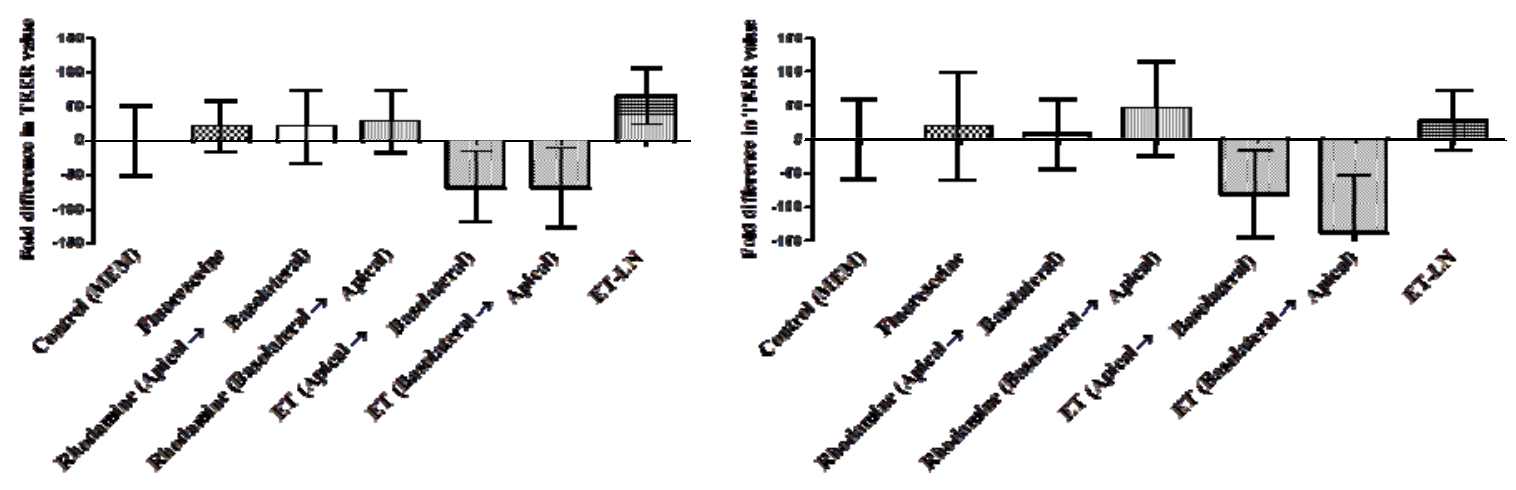

Figure 6. Fold increase/decrease in TEER value of Caco-2 monolayer (left) and Caco-2/Raji co-culture (right) after 2 hours of incubation with the different treatments. Free drug was the only treatment that produced a slight decrease in TEER values.

Rhodamine 123 and fluorescein were used as controls for the transport study. Rhodamine is a Pglycoprotein (P-gp) substrate, a multidrug efflux transporter that avoids the absorption of potentially risk molecules in the intestine [23]. As ET is also a P-gp substrate [17], rhodamine 123 was used to assess the P-gp function in Caco-2 in vitro intestinal model in order to further evaluate P-gp efflux transporter. Rhodamine 123 transport is performed by paracellular transport from the apical side while it is internalized by the transcellular route and eluded to the apical side by the P-gp in case of secretory transport [24]. Fluorescein is transported via paracellular route and is a common marker used in transport studies to assess the integrity of the tight junctions. Due to its size, fluorescein presents very low permeability in Caco-2 monolayers because of the presence of tight junctions. Table 1 shows the apparent permeabilities $\left(\mathrm{P}_{\mathrm{app}}\right)$ of both markers in the intestinal models studied. $\mathrm{P}_{\mathrm{app}}$ coefficients showed differences between the two culture models in case of secretory transport of rhodamine (from the basal to the apical compartment). As has been previously explained, the transport of both substances across Caco-2 monolayer occurs via the paracellular route, which is consistent with the low $\mathrm{P}_{\text {app }}$ absorptive coefficients of fluorescein and rhodamine. It means that tight junctions between cells prevented the crossing of these molecules. Regarding the differences in rhodamine transport in each culture, rhodamine secretory transport (from the basolateral to the apical side) was about 11.5 (Caco-2 monoculture) and 2.2 (Caco2/Raji B co-culture) times higher than absorptive transport. This difference in transport rates has been previously described by other authors [24]. The difference in rhodamine secretory transport was clearer in the case of the monoculture due to the larger presence of enterocyte-like cells which express the P-gp transporter. 
Table 1. Apparent permeability coefficient (Papp x 10-6 (cm/s) of fluorescein and rhodamine in Caco-2 and Caco-2/Raji B cultures **p $<0.01 ; * *$ * $<0.001$ vs. corresponding control group by two-way ANOVA (Bonferroni post-test).

\begin{tabular}{|c|c|c|}
\hline & Caco-2 monoculture & Caco-2/Raji B co-culture \\
\hline Fluorescein & $5.25 \pm 0.10$ & $6.05 \pm 4.38$ \\
\hline Rhoamine 123 (Apical) & $2.59 \pm 0.26$ & $9.04 \pm 3.49$ \\
\hline Rhodamine 123 (Basal) & $29.93 \pm 2.15$ & $19.83 \pm 0.53[* *$ \\
\hline
\end{tabular}

LN have been shown to increase the oral bioavailability of many different drugs including ET [3], and therefore many researchers have shown an interest in in vitro models of the intestinal barrier that make it easier to assess the absorption of these nanosystems at the intestinal level [10, 25]. Roger et al. [25] studied the permeability of Paclitaxel across Caco-2 monolayers, concluding that $\mathrm{LN}$ were able to increase drug uptake up to 3.5 times. Beloqui et al. [10] also conclude that LN enhance the absorption of saquinavir; however, they report a lower drug permeability of LN across Caco-2/Raji B co-culture than in the enterocyte-like model. With these premises we expected to observe increased permeability of ET-LN compared to the free ET in the Caco-2 in vitro intestinal model. Fig. 7 shows the amount of drug after the experiment in each compartment (apical and basolateral sides and cells) at the end of the experiment (2 hours). As can be observed, there was no ET in the basolateral side. This drawback did not allow us to calculate $\mathrm{P}_{\text {aap }}$ of each treatment. Considering that only part of the ET quantity that decreases from the apical compartment is quantified inside the cells, ET might suffer some metabolic process inside Caco-2 cells. ET is a synthetic PAF analog which contains a methyl group, attached by an ether linkage, at the sn2 position. This change in the molecule is related to a longer in vivo half-life because it prevents the degradation by the Phospholipase $\mathrm{A}_{2}\left(\mathrm{PLA}_{2}\right)$. However, ET is susceptible of being degraded by three pathways: i) cleavage of the alkyl group by glyceryl-ether monooxygenase (GEMO), ii) hydrolysis by Phospholipase C (PLC), and iii) Phospholipase D (PLD) hydrolysis [26]. Nowadays, ET degradation by GEMO has been discarded [26]. Wilcox et al [27] showed that ET is metabolized in MDCK, K-562 and HL-60 cells at very slow rates; however, hepatocytes metabolize up to half the administered ET after 24 hours of incubation [28]. In conclusion, as has been demonstrated in hepatocytes, ET might be absorbed and metabolized in enterocytes. This process could be one of the causes of its poor oral bioavailability [2], and so the fate of ET in enterocytes should be further studied. 

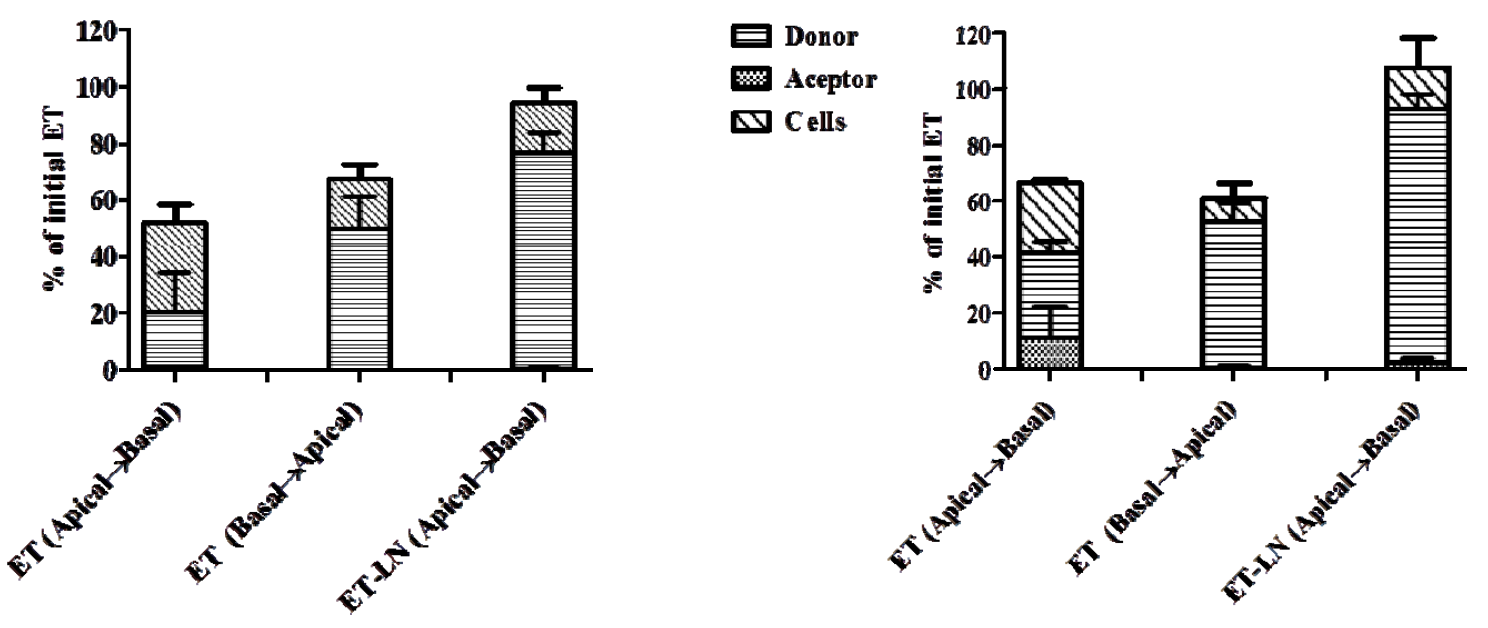

Figure 7. Edelfosine (\% of initial drug quantity on the donor compartment) present in the donor, acceptor and cell samples at the end of the transport experiment ( 2 hours of incubation) in Caco-2 monoculture (left) and Caco-2 co-culture (right). Free drug was not detectable in the basolateral chamber. It can also be observed that ET is internalized more rapidly than ET-LN in both culture models.

Nevertheless, in spite of being unable to calculate $\mathrm{P}_{\text {app }}$ `s coefficients, we were able to compare the internalization rates of ET and ET-LN in both in vitro intestinal models. ET was internalized into the cells very quickly in both culture models; besides, its internalization was higher from the apical than from the basolateral side. This might be explained by the lipid nature of ET; actually, hydrophobic compounds are taken up more efficiently by Caco-2 cells than hydrophilic molecules [10, 29]. This could mean that the concentration of ET used in the transport studies is high enough to saturate the P-gp efflux system. This saturation process, dependent on the drug concentration, has been previously demonstrated with other drugs [30]. Besides, the apical enterocyte membrane is enriched with lipid rafts, microdomains enriched with cholesterol and glycolipids [31,32] where ET accumulates in cells [33]. This high proportion of lipid rafts in the apical enterocyte membrane might also induce high absorption of the drug by the cells. Comparing both models, ET was internalized at a higher rate in both in vitro models: in fact, $76 \%$ and 90.96\% of LN remained in the apical side of Caco-2 monoculture and Caco-2/Raji co-culture respectively at the end of the transport experiment. These results are in agreement with results obtained by Roger et al. [25] who did not observe an increment in LN transport in the Caco-2/Raji B model in comparison with Caco-2 monoculture model. Taking into consideration that previous in vivo studies show an accumulation of drugs in the lymphatic system after their oral administration in LN [3], Caco-2/Raji co-culture might be not comparable to Peyer's patch follicle-associated epithelium (FAE) in the intestine. In this regard, the differentiation of induced pluripotent stem cells (iPS cells) into M cells $[34,35]$ has been documented. On the other hand, it seems clear the necessity of using bioreactors to achieve 3-D intestinal models [16]. In 
this work, the authors state the formation of a more reliable 3D intestinal in vitro model when Caco2 cells are incubated with primary isolated human microvascular endothelial cells (hMECs) on decellularized porcine jejunal segments, within a custom-made dynamic bioreactor system. However, despite the successful obtained results, the early stage of development of these approaches entails disadvantages in comparison to the classic well-defined Caco-2 intestinal models. Therefore, efforts in research should be focused on the further development of these complex models with the aim of improving the in vitro testing of the intestinal absorption of drugs.

\section{Conclusions}

ET is internalized at a higher rate in its free form in both in vitro intestinal models (Caco-2 and Caco2/Raji). In fact, about $76 \%$ and $90 \%$ of the initial LN quantity remains in the donor compartment in Caco2 monoculture and Caco-2/Raji co-culture respectively after 2 hours of incubation These results do not correlate with those observed in vivo when ET-LN were administered orally in mice, which suggests that the microfold model is not a good model to study the absorption of ET-LN across the intestinal barrier in vitro. Indeed, our results suggest that ET might suffer some metabolic process upon being internalized in Caco-2 and Caco-2/Raji intestinal models. The absence of drug in the acceptor compartment and its poor oral bioavailability might be explained by a similar metabolic process in enterocytes. Finally, the rapid ET internalization in the culture monolayers (about $80 \%$ of the initial ET in two hours) might be related to the hydrophilic nature of the drug, the elevated presence of lipid rafts in the apical surface of enterocytes and the saturation of the P-gp transporter. 


\section{Conflicts of interest}

The authors declare that they have no conflicts of interest in the research

\section{Acknowledgements}

This work has been carried out in the framework of the COST Action TD1004. Financial support from Caja Navarra Foundation, University of Navarra (FUN), the Government of Navarra, Department of Health (ref: 63/09, 'Ortiz de Landázuri’ fellowship) and the Spanish Ministry of Science and Innovation (SAF2010-15547) are acknowledged. B. Lasa-Saracibar is supported by the research grant from “Asociación de Amigos de la Universidad de Navarra". 


\section{References}

[1] van Blitterswijk, W. J.; Verheij, M. Anticancer mechanisms and clinical application of alkylphospholipids. Biochim Biophys Acta, 2013, 1831(3), 663-674.

[2] Estella-Hermoso de Mendoza, A.; Campanero, M. A.; de la Iglesia-Vicente, J.; Gajate, C.; Mollinedo, F.; Blanco-Prieto, M. J. Antitumor alkyl ether lipid edelfosine: tissue distribution and pharmacokinetic behavior in healthy and tumor-bearing immunosuppressed mice. Clin Cancer Res, 2009, 15(3), 858-864.

[3] Lasa-Saracibar, B.; Estella-Hermoso de Mendoza, A.; Guada, M.; Dios-Vieitez, C.; Blanco-Prieto, M. J. Lipid nanoparticles for cancer therapy: state of the art and future prospects. Expert Opin Drug Deliv, 2012, $9(10), 1245-1261$.

[4] Mollinedo, F.; de la Iglesia-Vicente, J.; Gajate, C.; Estella-Hermoso de Mendoza, A.; Villa-Pulgarin, J. A.; Campanero, M. A.; Blanco-Prieto, M. J. Lipid raft-targeted therapy in multiple myeloma. Oncogene, 2010, 29(26), 3748-3757.

[5] Estella-Hermoso de Mendoza, A.; Rayo, M.; Mollinedo, F.; Blanco-Prieto, M. J. Lipid nanoparticles for alkyl lysophospholipid edelfosine encapsulation: development and in vitro characterization. Eur $J$ Pharm Biopharm, 2008, 68(2), 207-213.

[6] Sambuy, Y.; De Angelis, I.; Ranaldi, G.; Scarino, M. L.; Stammati, A.; Zucco, F. The Caco-2 cell line as a model of the intestinal barrier: influence of cell and culture-related factors on Caco-2 cell functional characteristics. Cell Biol Toxicol, 2005, 21(1), 1-26.

[7] Mabbott, N. A.; Donaldson, D. S.; Ohno, H.; Williams, I. R.; Mahajan, A. Microfold (M) cells: important immunosurveillance posts in the intestinal epithelium. Mucosal Immunol, 2013, 6(4), 666-677.

[8] Antunes, F.; Andrade, F.; Araujo, F.; Ferreira, D.; Sarmento, B. Establishment of a triple co-culture in vitro cell models to study intestinal absorption of peptide drugs. Eur J Pharm Biopharm, 2012, 83(3), $427-435$. 
[9] des Rieux, A.; Fievez, V.; Theate, I.; Mast, J.; Preat, V.; Schneider, Y. J. An improved in vitro model of human intestinal follicle-associated epithelium to study nanoparticle transport by M cells. Eur J Pharm Sci, 2007, 30(5), 380-391.

[10] Beloqui, A.; Solinis, M. A.; Gascon, A. R.; del Pozo-Rodriguez, A.; des Rieux, A.; Preat, V. Mechanism of transport of saquinavir-loaded nanostructured lipid carriers across the intestinal barrier. $J$ Control Release, 2012, 166(2), 115-123.

[11] Kerneis, S.; Bogdanova, A.; Kraehenbuhl, J. P.; Pringault, E. Conversion by Peyer's patch lymphocytes of human enterocytes into M cells that transport bacteria. Science, 1997, 277(5328), 949952.

[12] Lo, D.; Tynan, W.; Dickerson, J.; Scharf, M.; Cooper, J.; Byrne, D.; Brayden, D.; Higgins, L.; Evans, C.; O'Mahony, D. J. Cell culture modeling of specialized tissue: identification of genes expressed specifically by follicle-associated epithelium of Peyer's patch by expression profiling of Caco-2/Raji cocultures. Int Immunol, 2004, 16(1), 91-99.

[13] Ander Estella-Hermoso de Mendoza, M. J. B.-P., Miguel Angel Campanero, Faustino Mollinedo, Janny Villa-Pulgarín, Rubén Varela. Development and use of lipidic nanoparticles loaded with edelfosine and ether phospholipids in antitumoral and antiparasitic therapy. Spain. Patent P201130433, October, 03, 2011.

[14] Estella-Hermoso de Mendoza, A.; Campanero, M. A.; Mollinedo, F.; Blanco-Prieto, M. J. Comparative study of A HPLC-MS assay versus an UHPLC-MS/MS for anti-tumoral alkyl lysophospholipid edelfosine determination in both biological samples and in lipid nanoparticulate systems. J Chromatogr B Analyt Technol Biomed Life Sci, 2009, 877(31), 4035-4041.

[15] Millicell ${ }^{2}$ ERS-2 User Guide.

http://www.millipore.com/userguides.nsf/a73664f9f981af8c852569b9005b4eee/a5708d8207d7accf85257 609005c7c5f/\$FILE/00108103.pdf (Accesed Sept 11, 2013).

[16] Pusch, J.; Votteler, M.; Gohler, S.; Engl, J.; Hampel, M.; Walles, H.; Schenke-Layland, K. The physiological performance of a three-dimensional model that mimics the microenvironment of the small intestine. Biomaterials, 2011, 32(30), 7469-7478. 
[17] Estella-Hermoso de Mendoza, A.; Preat, V.; Mollinedo, F.; Blanco-Prieto, M. J. In vitro and in vivo efficacy of edelfosine-loaded lipid nanoparticles against glioma. J Control Release, 2011, 156(3), 421426.

[18] Lasa-Saracibar, B.; Estella-Hermoso de Mendoza, A.; Mollinedo, F.; Odero, M. D.; Blanco-Prieto, M. J. Edelfosine lipid nanosystems overcome drug resistance in leukemic cell lines. Cancer Lett, 2013, $334(2), 302-310$.

[19] Gullberg, E.; Leonard, M.; Karlsson, J.; Hopkins, A. M.; Brayden, D.; Baird, A. W.; Artursson, P. Expression of specific markers and particle transport in a new human intestinal M-cell model. Biochem Biophys Res Commun, 2000, 279(3), 808-813.

[20] Stevenson, B. R.; Siliciano, J. D.; Mooseker, M. S.; Goodenough, D. A. Identification of ZO-1: a high molecular weight polypeptide associated with the tight junction (zonula occludens) in a variety of epithelia. J Cell Biol, 1986, 103(3), 755-766.

[21] Kerneis, S.; Bogdanova, A.; Colucci-Guyon, E.; Kraehenbuhl, J. P.; Pringault, E. Cytosolic distribution of villin in M cells from mouse Peyer's patches correlates with the absence of a brush border. Gastroenterology, 1996, 110(2), 515-521.

[22] Weston, S. A.; Parish, C. R. New fluorescent dyes for lymphocyte migration studies. Analysis by flow cytometry and fluorescence microscopy. J Immunol Methods, 1990, 133(1), 87-97.

[23] Xue, X.; Liang, X. J. Overcoming drug efflux-based multidrug resistance in cancer with nanotechnology. Chin J Cancer, 2012, 31(2), 100-109.

[24] Troutman, M. D.; Thakker, D. R. Rhodamine 123 requires carrier-mediated influx for its activity as a P-glycoprotein substrate in Caco-2 cells. Pharm Res, 2003, 20(8), 1192-1199.

[25] Roger, E.; Lagarce, F.; Garcion, E.; Benoit, J. P. Lipid nanocarriers improve paclitaxel transport throughout human intestinal epithelial cells by using vesicle-mediated transcytosis. J Control Release, 2009, 140(2), 174-181.

[26] Gajate, C.; Mollinedo, F. Biological activities, mechanisms of action and biomedical prospect of the antitumor ether phospholipid ET-18-OCH(3) (edelfosine), a proapoptotic agent in tumor cells. Curr Drug Metab, 2002, 3(5), 491-525. 
[27] Wilcox, R. W.; Wykle, R. L.; Schmitt, J. D.; Daniel, L. W. The degradation of platelet-activating factor and related lipids: susceptibility to phospholipases C and D. Lipids, 1987, 22(11), 800-807.

[28] Magistrelli, A.; Villa, P.; Benfenati, E.; Modest, E. J.; Salmona, M.; Tacconi, M. T. Fate of 1-Ooctadecyl-2-O-methyl-rac-glycero-3-phosphocholine (ET18-OME) in malignant cells, normal cells, and isolated and perfused rat liver. Drug Metab Dispos, 1995, 23(1), 113-118.

[29] Gaumet, M.; Gurny, R.; Delie, F. Interaction of biodegradable nanoparticles with intestinal cells: the effect of surface hydrophilicity. Int J Pharm, 2009, 390(1), 45-52.

[30] Jang, S. H.; Wientjes, M. G.; Au, J. L. Kinetics of P-glycoprotein-mediated efflux of paclitaxel. J Pharmacol Exp Ther, 2001, 298(3), 1236-1242.

[31] Danielsen, E. M.; Hansen, G. H. Lipid rafts in epithelial brush borders: atypical membrane microdomains with specialized functions. Biochim Biophys Acta, 2003, 1617(1-2), 1-9.

[32] Kunding, A. H.; Christensen, S. M.; Danielsen, E. M.; Hansen, G. H. Domains of increased thickness in microvillar membranes of the small intestinal enterocyte. Mol Membr Biol, 2010, 27(4-6), 170-177.

[33] Ausili, A.; Torrecillas, A.; Aranda, F. J.; Mollinedo, F.; Gajate, C.; Corbalan-Garcia, S.; de Godos, A.; Gomez-Fernandez, J. C. Edelfosine is incorporated into rafts and alters their organization. $J$ Phys Chem B, 2008, 112(37), 11643-11654.

[34] Iwao T., Toyota M, Miyagawa Y., Okita H., Kiyokawa N., Akutsu H., Umezawa A., Nagata K., Matsunaga T. Differentiation of human induced pluripotent stem cells into functional enterocyte-like cells using a simple method. Drug Metab Pharmacok, 2013 (http://dx.doi.org/10.2133/dmpk.DMPK-13-RG005).

[35] McCracken KW., Howell JC., Wells JM., Spence JR. Generating human intestinal tissue from pluripotent stem cells in vitro. Nat Protoc, 2011, 6(12),1920-1928. 